\title{
Citrulline/ malate promotes aerobic energy production in human exercising muscle
}

\author{
D Bendahan, J P Mattei, B Ghattas, S Confort-Gouny, M E Le Guern, PJ Cozzone
}

Br J Sports Med 2002;36:282-289

See end of article for authors' affiliations

$\ldots \ldots \ldots \ldots \ldots \ldots$.

Correspondence to:

Professor Cozzone, Centre

de Résonance Magnétique

Biologique et M édicale,

UMR CN RS 6612, Faculté

de $M$ édecine de Marseille,

27, Bd J Moulin, 13005

$M$ arseille France;

patrick.cozzone@medecine.

univ-mrs.fr

Accepted

15 February 2002
Background: Previous studies have shown an antiasthenic effect of citrulline/ malate (CM) but the mechanism of action at the muscular level remains unknown.

Objective: To investigate the effects of CM supplementation on muscle energetics.

Methods: Eighteen men complaining of fatigue but with no documented disease were included in the study. A restexercise (finger flexions)-recovery protocol was performed twice before (D-7 and D0), three times during (D3, D8, D15), and once after (D22) 15 days of oral supplementation with $6 \mathrm{~g} /$ day $\mathrm{CM}$. M etabolism of the flexor digitorum superficialis was analysed by ${ }^{31} \mathrm{P}$ magnetic resonance spectroscopy at $4.7 \mathrm{~T}$.

Results: M etabolic variables measured twice before $\mathrm{CM}$ ingestion showed no differences, indicating good reproducibility of measurements and no learning effect from repeating the exercise protocol. CM ingestion resulted in a significant reduction in the sensation of fatigue, a $34 \%$ increase in the rate of oxidative ATP production during exercise, and a $20 \%$ increase in the rate of phosphocreatine recovery after exercise, indicating a larger contribution of oxidative A TP synthesis to energy production. Considering subjects individually and variables characterising a erobic function, extrema were measured after either eight or 15 days of treatment, indicating chronological heterogeneity of treatment induced changes. 0 ne way analysis of variance confirmed improved aerobic function, which may be the result of an enhanced malate supply activating ATP production from the tricarboxylic acid cycle through anaplerotic reactions.

Conclusion: The changes in muscle metabolism produced by CM treatment indicate that CM may promote aerobic energy production.

\footnotetext{
$\mathrm{C}$
} itrulline/malate (CM; Stimol; CAS 54940-97-5) is a mixture of citrulline, which is involved in the urea cycle, and malate, a tricarboxylic acid cycle (TCA) intermediate. It is usually prescribed as an antiasthenic treatment. Previous studies in humans and rats have indicated that $\mathrm{CM}$ treatment improves muscle performance of subjects suffering from asthenia. Several double blinded versus placebo clinical investigations have clearly shown an antiasthenic effect of CM under various conditions of fatigue. ${ }^{12}$ From a systemic point of view, measurements of lactate and ammonia performed during and after bicycle exercise have shown increased rates of clearance from the blood after exercise ${ }^{3}{ }^{4}$ as the result of CM treatment, and an effect on acid-base balance has been reported at hepatic and renal levels. ${ }^{5}$ Experiments using cellular models have suggested an action of CM on lactate metabolism. ${ }^{6}$ Clinically, CM treatment has been shown to improve recovery of physical activity after acute diseases. ${ }^{1}$ Although all these results indicate potential effects of $\mathrm{CM}$ on muscle performance, the actual effect remains unknown. In a study of muscle fatigue induced by bacterial endotoxins in a rat model, it has been shown that mechanical characteristics - that is, resistance to fatigue-improved after $\mathrm{CM}$ treatment. This beneficial effect has been hypothetically linked to nitric oxide synthesis through the production of citrulline. ${ }^{31} \mathrm{P}$ magnetic resonance spectroscopy (MRS) is now well established as a non-invasive method for measuring metabolic changes before, during, and after muscular exercise. It provides kinetic information on high energy phosphate compounds and intracellular $\mathrm{pH}$ and has been used to document normal muscle metabolism, ${ }^{8-11}$ metabolic effects of training, ${ }^{12}{ }^{13}$ and metabolic deviations in various metabolic myopathies. ${ }^{14-16}$ The purpose of our study was to document directly the effect of CM, if any, on muscle energetics in humans by using ${ }^{31} \mathrm{P} M \mathrm{MS}$.

\section{METHODS}

Approach to the problem

The antiasthenic effect of $\mathrm{CM}$, which has been widely reported in humans and animals ${ }_{1}{ }^{-7}$ may be mediated by either citrulline or malate or both. Acceleration of the clearance of plasma ammonium and lactate in humans has been linked to the role of citrulline in the urea cycle. On the other hand, malate, an intermediate of the TCA cycle, may be involved in the beneficial effects on recovery from physical fatigue in humans. In this study, we hypothesised that the antiasthenic effects of CM may be due to changes affecting muscle energetics. These potential changes are amenable to measurement by ${ }^{31} \mathrm{P} M R S, a$ non-invasive technique allowing continuous measurement of high energy phosphate compounds and $\mathrm{pH}$ during transition from rest to exercise and during the recovery period. Ultimately, this method provides quantitative information on the contributions of aerobic and anaerobic pathways to energy production, which may be affected by CM supplementation. To test our hypothesis, we investigated muscle energetics in subjects in an acute phase of fatigue. Subjects were selected according to CM prescription-that is, if they complained of fatigue but with no documented disease. Clinical examination, electrocardiography, and blood tests were performed to eliminate any disease that could account, directly or indirectly, for the fatigue.

\section{Subjects}

Eighteen sedentary male subjects (mean (SD) age 31.1 (9.2)) who complained of fatigue were enrolled in the study.

Abbreviations: CM, citrulline/ malate; TCA, tricarboxylic acid cycle; MRS, magnetic resonance spectroscopy; PCr, phosphocreatine; EC, energy cost 
Fatigue was characterised as described by $\mathrm{M}$ ac Nelley ${ }^{17}$ and by visual analogue scales. ${ }^{18} \mathrm{M}$ ean (SD) height and weight were respectively $177.6(4.6) \mathrm{cm}$ and $72.7(10.6) \mathrm{kg}$. M ean (SD) scores were 6 (0.9) and 49 (15) for Mac Nelley and visual analogue scales respectively. Before inclusion, all subjects gave their informed written consent, and health status was assessed from medical history, physical examination, and blood tests. None of the subjects were taking any medication at the time of the study, and they did not suffer from any diseases. The study was approved by the ethics committee of the Timone Hospital in M arseille.

\section{${ }^{31} \mathrm{P}$ MRS}

${ }^{31} \mathrm{P}$ M RS exploration of finger flexor muscles was carried out as previously described using a Bruker $47 / 30$ Biospec spectrometer interfaced with a $30 \mathrm{~cm}$ bore, $4.7 \mathrm{~T}$ horizontal superconducting magnet. ${ }^{19}$ The protocol was explained in detail to each patient before the exploration. Subjects sat on a chair by the magnet with their dominant arm resting in the magnet bore. To ensure good venous return, the forearm was placed at about the same height as the shoulder. M agnetic field homogeneity was optimised by monitoring the signal from the water and lipid protons at $200.14 \mathrm{M} \mathrm{Hz}$. Pulsing conditions ( 1.8 second interpulse delay, $120 \mu$ s pulse length) were chosen to optimise the ${ }^{31} \mathrm{P}$ M RS signal obtained with a $50 \mathrm{~mm}$ diameter, double tuned surface coil positioned over the flexor digitorum superficialis muscle. Spectra were time averaged over one minute (32 scans) and sequentially recorded over three minutes of rest, three minutes of exercise, and 20 minutes of recovery. Exercise consisted of finger flexions performed at 1.5 second intervals lifting a $6 \mathrm{~kg}$ weight as described previously. ${ }^{9}$ All subjects were able to complete the exercise. The three minute exercise duration and intensity were chosen to ensure appreciable decreases in $\mathrm{pH}$ and phosphocreatine $(\mathrm{PCr})$ in all subjects and the completion of the protocol. The sliding of the weight was recorded using a displacement transducer connected to a personal computer. Force was measured using ATS software (Sysma, France), and power output was calculated for each minute of exercise. This protocol was repeated twice before ( $D-7$ and $D 0)$, three times during (D3, $D 8, D 15)$, and once after (D22) oral administration of CM. The dose was two ampoules ( $1 \mathrm{~g} \mathrm{CM}$ per ampoule) three times a day for 15 days (from D1 to D15). CM was provided by Biocodex ( $M$ ontrouge, France). The concentrations of citrulline and malate in each ampoule were 5.5 and $4.7 \mathrm{~g} / 100 \mathrm{ml}$ respectively. The purity of the product was greater than $99 \%$. Resource and time constraints meant that 18 subjects ( 108 separate experiments) were the most we could recruit.

\section{Data analysis}

Raw MRS signals were transferred to an IBM RISC 6000 workstation and processed using the NMR1 spectroscopy software (New Methods Research Inc, Syracuse, New York, USA). Fourier transform and deconvolution of free induction decays corresponding to a line broadening of $15 \mathrm{~Hz}$ were then performed. Baseline correction was obtained with baseline deconvolution using computer estimated filter size and flattening factors. ${ }^{20}$ The area of each resonance was measured by curve fitting of the spectrum to Lorentzian lines. Concentrations of $\mathrm{PCr}$, inorganic phosphate $\left(\mathrm{P}_{\mathrm{i}}\right), \mathrm{ATP}$, and phosphomonoesters were calculated. Values were corrected for differential magnetic saturation and expressed as absolute concentrations relative to the ATP concentration found at rest in muscle biopsy specimens $(8.2 \mathrm{mmol} / \mathrm{l}$ intracellular water $(\mathrm{mM})){ }^{21}$. Free cytosolic ADP concentration was calculated from the creatine kinase equilibrium, assuming a total creatine content of $42.5 \mathrm{mM}$, which is usually reported for muscle biopsy specimens. ${ }^{8}$ Intracellular $\mathrm{pH}$ was calculated from the chemical shift of $\mathrm{P}_{1}$ relative to $\mathrm{PCr}^{22}$

As a standardisation procedure, the magnitude of changes in $[\mathrm{PCr}]$ and $\mathrm{pH}$ measured at the end of exercise were scaled to power output and referred to as $\Delta[\mathrm{PCr}] /$ power and $\Delta \mathrm{pH} /$ power respectively. Taking into account all the data points, the rate of $\mathrm{PCr}$ resynthesis during the recovery period was fitted to a first order exponential equation as follows:

$$
[P C r](t)=[P C r]_{\text {rest }}+[P C r]_{\text {cons }}\left(\exp ^{-k t}\right)
$$

where $t$ is time, and $[\mathrm{PCr}]_{\text {rest }}$ and $[\mathrm{PCr}]_{\text {cons }}$ respectively indicate concentration of $\mathrm{PCr}$ at rest and extent of $\mathrm{PCr}$ consumed at end of exercise. The rate constant ( $\mathrm{k}$ ) is expressed in $\mathrm{min}^{-1}$. The initial rate of $\mathrm{PCr}$ resynthesis was calculated as the product of $\mathrm{k}$ and $[\mathrm{PCr}]_{\text {cons }}$ resulting from the time zero derivative of equation $(1){ }^{23}$

Quantitative analysis of metabolic changes

Rates of aerobic and anaerobic ATP production were calculated in exercising muscle as originally described by Kemp \& al. ${ }^{24}$ Briefly, the energy cost (EC) of contraction was calculated at the onset of exercise, the oxidative contribution being regarded as negligible at that time. ${ }^{10}$ Rates of glycogenolytic ATP production were calculated taking into account changes in $[\mathrm{PCr}]$ and $\mathrm{pH}$ during exercise, in addition to buffering capacity ${ }^{25}$ and proton efflux. ${ }^{26}$ Assuming that EC remains proportional to power output throughout the exercise period, rates of aerobic ATP production can be calculated at any time during exercise as the difference between EC and anaerobic ATP production scaled to power output. ${ }^{24}$

Statistical analysis

The General Linear Models procedure of the SAS software (options REPEATED, LSM EANS, and CONTRAST) was used to perform an analysis of variance.

For variables measured during exercise (therefore changing with time) such as metabolite concentration, $\mathrm{pH}$, power output, and rates of ATP production, two way analysis of variance with repeated measures (repeated factors being exercise and treatment duration) were used. Wilk's $\lambda$ tests were performed to analyse the effects of exercise duration and the interaction between exercise and treatment duration. Post hoc repeated comparisons (Scheffe's contrasts) were performed to test specific hypotheses.

For rates of recovery of [PCr] and $\mathrm{pH}$, one way analysis of variance with repeated measures (repeated factor being treatment duration) was used. $\mathrm{F}$ tests were performed to determine the overall effect of treatment duration on the recovery kinetics. Then, multiple comparison procedures (Scheffe's contrasts) were used to compare each value with the corresponding value measured before and after the treatment. $p<0.05$ was considered significant. Homogeneity of variances and normality of residuals were checked using Levene's test, an option of General Linear M odels for homogeneity of stack variances and normality of residuals. When considering repeated measurements, analysis of variance type methods are the only appropriate statistical methods. Given that variance of each measurement is taken into account, these methods are usually more powerful than simple t tests, which are actually not reliable for multiple repeated measurements.

\section{RESULTS}

Metabolic changes and reproducibility of results

Table 1 summarises intracellular metabolite concentrations and $\mathrm{pH}$ calculated at rest; they do not differ from results reported previously. ${ }^{8}$ Figure 1 shows metabolic changes recorded during the rest-exercise-recovery protocols and before the treatment. Clearly, no significant changes were observed between the two measurements performed a week apart before the treatment. Exerciseled to PCr consumption as a result of ATP hydrolysis by the myosin ATPases, illustrating the buffering effect of PCr on ATP concentration (fig 1A). 
Table 1 Magnetic resonance spectroscopy variables measured before treatment with citrulline/ malate (D-7 and D0)

\begin{tabular}{|c|c|c|c|}
\hline & $\mathrm{D}-7$ & D0 & p Value \\
\hline \multicolumn{4}{|l|}{ Rest } \\
\hline$[\mathrm{PCr}](\mathrm{mM})$ & $37.5(0.3)$ & $38.2(0.8)$ & 0.23 \\
\hline $\mathrm{pH}$ & $6.99(0.01)$ & $7.01(0.01)$ & 0.69 \\
\hline \multicolumn{4}{|l|}{ Exercise } \\
\hline \multicolumn{4}{|l|}{ Initial phase of exercise } \\
\hline $\mathrm{EC}(\mathrm{mM} / \mathrm{min} / \mathrm{W})$ & $22.5(2.1)$ & $22.9(1.8)$ & 0.85 \\
\hline $\mathrm{Vi}_{\mathrm{ex}}(\mathrm{mM} / \mathrm{min})$ & $38.4(4.5)$ & $42.5(5.4)$ & 0.41 \\
\hline \multicolumn{4}{|l|}{ End of exercise } \\
\hline $\operatorname{PCr}(\%)$ & $61.4(3.8)$ & $66(4.5)$ & 0.69 \\
\hline $\mathrm{pH}$ & $6.52(0.04)$ & $6.47(0.6)$ & 0.39 \\
\hline Power (W) & $1.55(0.1)$ & $1.61(0.1)$ & 0.54 \\
\hline$\Delta[\mathrm{PCr}] /$ power (mM PCr/ W ) & $16.5(1.6)$ & $16.2(1.2)$ & 0.84 \\
\hline$\Delta \mathrm{pH} /$ power (pH unit/ W ) & $0.33(0.03)$ & $0.35(0.03)$ & 0.55 \\
\hline Rate of oxidative ATP production ( $\%$ EC) & $41.9(7)$ & $41.5(5)$ & 0.88 \\
\hline \multicolumn{4}{|l|}{ Recovery } \\
\hline $\mathrm{Vi}_{\text {rec }}(\mathrm{mM} / \mathrm{min})$ & $14.2(2.3)$ & $14.5(2.8)$ & 0.89 \\
\hline \multicolumn{4}{|c|}{ 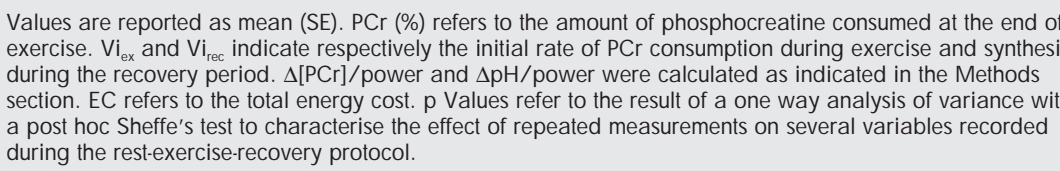 } \\
\hline
\end{tabular}
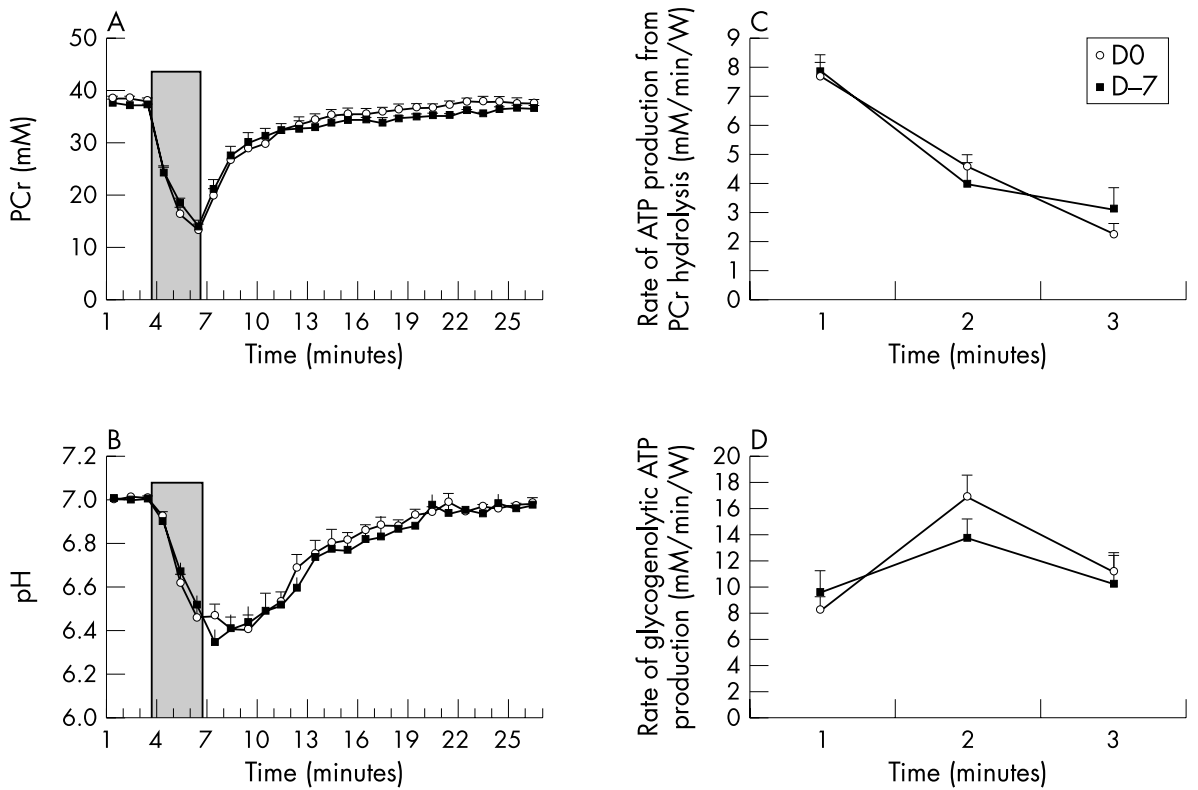

Figure 1 Time dependent changes in phosphocreatine $(\mathrm{PC})$ concentration $(\mathrm{A})$ and $\mathrm{pH}(\mathrm{B})$ during the restexercise-recovery protocol. Shaded area represents the three minute exercise. Rate of ATP production from PCr hydrolysis (C) and from glycogenolysis (D) during the exercise period. Results are presented as means with error bars representing $1 \mathrm{SE}$.

Cytosolic pH changes (fig 1B) reflect the balance between protons produced by glycogenolysis, consumed through the creatine kinase equilibrium, and handled by buffering components and transporters. A transient acidosis was observed at the onset of recovery as a result of proton production through the creatine kinase equilibrium. Recovery kinetics of $\mathrm{PCr}$ and $\mathrm{pH}$ remained similar before the treatment period (fig $1 \mathrm{~A}, \mathrm{~B}$ ). Rates of anaerobic ATP production were calculated taking account of changes in [PCr] and pH (fig 1C,D). As for changes in $[\mathrm{PCr}$ ] and $\mathrm{pH}$, no variation was measured between rates calculated at D-7 and D0. The overall rate of ATP turnover during exercise remained the same at D-7 and D0, showing a constant proportionality between energy production and mechanical performance between the two measurements (table 1). Similarly, the initial rate of PCr hydrolysis measured at the beginning of exercise, the final muscle energetic status after the three minute exercise, and the magnitude of metabolic changes scaled to power output were all identical at D-7 and D0 (table 1 and fig 1).

\section{Metabolic effects of treatment}

No adverse reactions to CM treatment were reported by the volunteers or observed by the doctors monitoring the clinical trial. The variables measured at rest, including [ $\mathrm{PCr}]$ and $\mathrm{pH}$, were similar during and after treatment, suggesting no effect of treatment on muscle energetic status at rest (table 2). Considering the group as a whole, work rate measured during the three minute exercise test remained constant during CM treatment, and the total rate of energy turnover did not change, indicating a constant proportionality between the rate of ATP consumption and mechanical output (table 2). Similarly, the rate of ATP production from PCr hydrolysis was constant throughout the treatment (fig 2A). In contrast, a 34\% increase in the rate of aerobic ATP production was observed, 
Table 2 Magnetic resonance spectroscopy variables measured before, during, and after treatment with citrulline/ malate

\begin{tabular}{|c|c|c|c|}
\hline & Before & During & After \\
\hline \multicolumn{4}{|l|}{ Rest } \\
\hline$[\mathrm{PCr}](\mathrm{mM})$ & $37.8(0.3)$ & $37.5(0.3)$ & $37.7(0.5)$ \\
\hline $\mathrm{pH}$ & $7(0.01)$ & $7(0.01)$ & $6.99(0.01)$ \\
\hline \multicolumn{4}{|l|}{ Exercise } \\
\hline \multicolumn{4}{|l|}{ Initial phase of exercise } \\
\hline $\mathrm{EC}(\mathrm{mM} / \mathrm{min} / \mathrm{W})$ & $22.8(2.1)$ & $19.6(1.6)$ & $21(1.7)$ \\
\hline $\mathrm{Vi}_{\mathrm{ex}}(\mathrm{mM} / \mathrm{min})$ & $46.7(5.3)$ & $57.2(3.9)$ & $41.9(4.4)$ \\
\hline \multicolumn{4}{|l|}{ End of exercise } \\
\hline $\mathrm{PCr}(\%)$ & $69(3.8)$ & $72(2)$ & $61(3)$ \\
\hline $\mathrm{pH}$ & $6.57(0.03)$ & $6.58(0.04)$ & $6.49(0.05)$ \\
\hline Power (W) & $1.7(0.1)$ & $2(0.1)^{*}$ & $1.7(0.08)$ \\
\hline $\mathrm{pH}$ & $6.57(0.03)$ & $6.58(0.04)$ & $6.49(0.05)$ \\
\hline$\Delta[\mathrm{PCr}] /$ power $(\mathrm{mM} \mathrm{PCr} / \mathrm{W})$ & $14.9(1.2)$ & $12.4(0.8)$ & $14.1(0.7)$ \\
\hline$\Delta \mathrm{pH} / \mathrm{power}(\mathrm{pH}$ unit/ W) & $0.29(0.05)$ & $0.24(0.02)^{*}$ & $0.3(0.03)$ \\
\hline Rate of oxidative ATP production ( $\% E C$ ) & $54(12)$ & $74(4)^{*}$ & $45(6)$ \\
\hline \multicolumn{4}{|l|}{ Recovery } \\
\hline $\mathrm{Vi}_{\text {rec }}(\mathrm{mM} / \mathrm{min})$ & $16.9(2.9)$ & $24.2(3.2)^{*}$ & $16.4(1.9)$ \\
\hline \multicolumn{4}{|c|}{$\begin{array}{l}\text { Values are reported as mean (SE). } \mathrm{PCr}(\%) \text { refers to the a mount of phosphocreatine consumed at the end of } \\
\text { exercise. } \mathrm{Vi}_{\text {ex }} \text { and } \mathrm{Vi}_{\text {rec }} \text { indicate respectively the initial rate of } \mathrm{PC} \text { consumption during exercise and synthesis } \\
\text { during the recovery period. } \Delta[\mathrm{PC} r] / \text { power and } \Delta \mathrm{pH} / \mathrm{pow} \text { er were calculated as indicated in the } \mathrm{M} \text { ethods } \\
\text { section. EC refers to the total energy cost. As indicated in the text, before and during refer to extrema of } \\
\text { variables calculated before and during the treatment. } \\
\text { * Significantly different from measurements before and after the treatment period. }\end{array}$} \\
\hline
\end{tabular}

reaching a maximum after three days of treatment and then slowly returning to the pretreatment value ( fig 2B). However, this $34 \%$ increase was not significant, although very close to the set threshold. The concentration of ADP, which is known to be the driving force of oxidative ATP synthesis, was not affected by $\mathrm{CM}$ treatment. Figure 3 shows the changes in [PCr] and $\mathrm{pH}$ measured throughout the experimental protocol and related to work output. Both variables decreased with increasing work output, and a shift towards higher values of work output for a given value of either $[\mathrm{PCr}]$ or $\mathrm{pH}$ was measured mainly at the end of exercise. Also, a 20\% increase was observed for the rate of [PCr] recovery, indicating more rapid aerobic ATP production at D8 than before treatment. Overall, a reduction in fatigue sensation was reported by all the subjects. On both scales, the effect was maximum after 15 days of treatment.

Looking at the data individually, a large heterogeneity was observed among the subjects, who displayed extrema after either eight or 15 days of treatment. This chronological heterogeneity of individual responses during CM treatment may prevent statistical significance from being reached for the whole group. In other words, because the chronological responses to the treatment differed among subjects, comparative analysis of the average values of a given variable on a given day may not be appropriate. Extrema of some variables, referring to maxima or minima between D8 and D15, were therefore calculated and compared, using a one way analysis of variance, with extrema measured before and after the treatment. The new occurrences were identified as "before" (corresponding to the extrema measured among two measurements before the treatment period), "during" (corresponding to the extrema measured among two measurements, D8 and D15, throughout the treatment period), and "after" (corresponding to measurement performed after the treatment period).

Overall, the results showed no treatment induced changes in the variables measured at rest and at the onset of exercise, indicating that neither the energetic status of the muscle nor the total rate of ATP turnover were modified after CM administration (table 2). In contrast, a significant decrease was measured for $\Delta \mathrm{pH} /$ power ( fig 3 and table 2). Also, significantly more aerobically produced ATP was measured during CM treatment (fig 4 and table 2), indicating a transient enhancement of oxidative processes, as suggested by the initial statis-
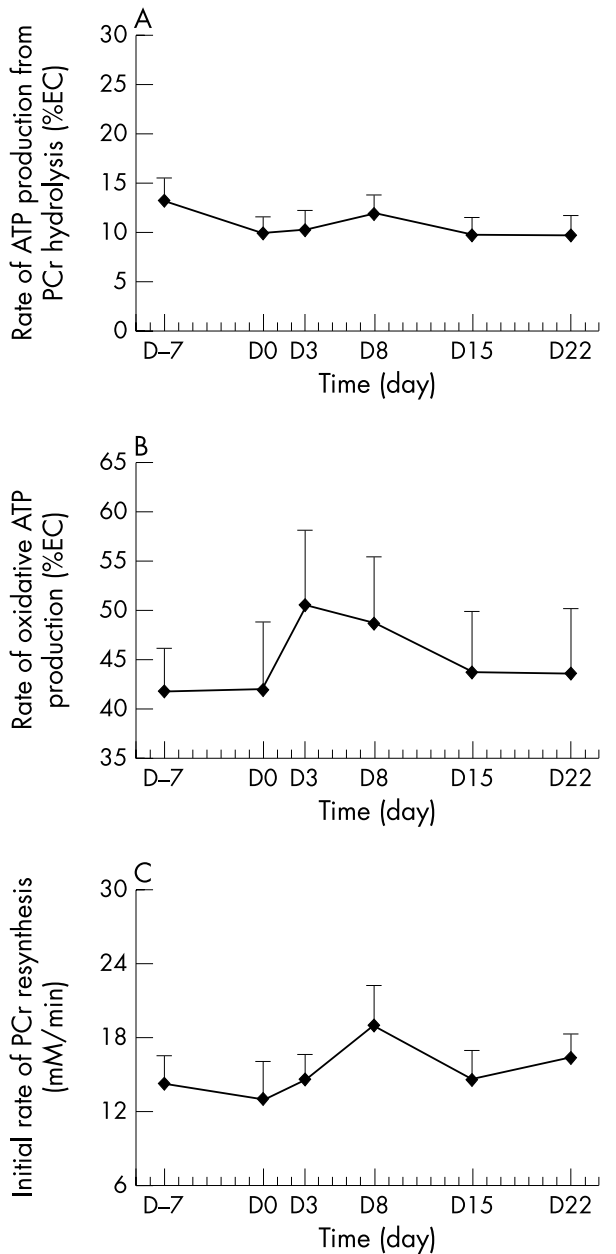

Figure 2 Time dependent changes in the rate of ATP production from phosphocreatine ( $\mathrm{PCr}$ ) hydrolysis (A) and the aerobic pathway (B) expressed as percentage of energy cost (\%EC). Evolution of the initial rate of $\mathrm{PC}$ resynthesis $(\mathrm{C})$ during the protocol. Results are presented as means with error bars representing $1 \mathrm{SE}$. 

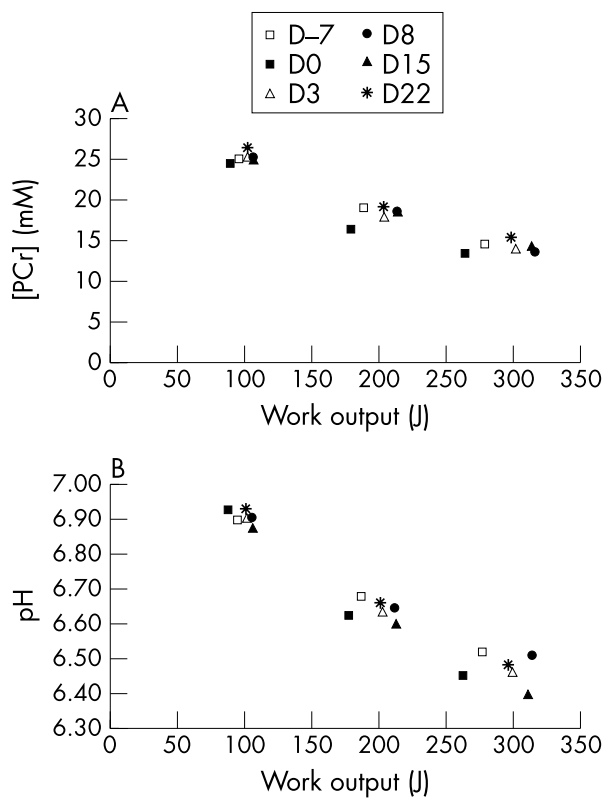

Figure 3 Changes in (A) phosphocreatine concentration ([PC r]) and (B) $\mathrm{pH}$ recorded as a function of work output during exercise. Error bars were omitted for the sake of clarity. A shift towards higher values of work output for both variables during treatment with citrulline/ malate indicates an improvement in muscle performance.

tical analysis. Further, the kinetics of [PCr] recovery after exercise were significantly faster after treatment (fig 4, table 2), illustrating the positive effect of $\mathrm{CM}$ on aerobic metabolism.

\section{DISCUSSION}

The main results of this study indicate that $\mathrm{CM}$ treatment results in a larger contribution of aerobic pathways to global energy production. This result must be analysed in the light of the high reproducibility and stability of metabolic indices recorded one week and one day before treatment. The stability of these measurements reflects the reliability of the method and the absence of a learning effect from repetition of the protocol. Given the stability and reproducibility of the measurements, no placebo control group was used.

\section{Methodological considerations}

Considering the spatial sel ectivity of M RS measurements performed with a surface coil, one could argue that the metabolic changes may be recorded from a mixture of activated and inactivated muscles, thereby limiting the relation between mechanical output and metabolic changes. Using T2 weighted magnetic resonance imaging experiments, we have previously shown, in agreement with others, that finger flexions using the four fingers (as in this experiment) is mainly associated with activation of the finger flexors (profundus and superficialis). Considering the size of our coil $(5 \mathrm{~cm})$, it is likely that metabolic changes were recorded from all the muscles producing force. Furthermore, if metabolic changes had been recorded from a mixture of activated and inactivated muscles, we would have observed a split $P_{i}$ resonance on the spectra because of various extents of acidosis in each muscle compartment. This was never the case, further suggesting, in agreement with the multiple control measurements performed at the start of the experiment, that the positive effect of CM treatment on oxidative metabolism was not the result of any experimental limitation.

It is still debated whether the kinetics of $\mathrm{PCr}$ recovery are monoexponential or biexponential. We chose to use a widely reported and accepted method using all data points ${ }^{27}$ and
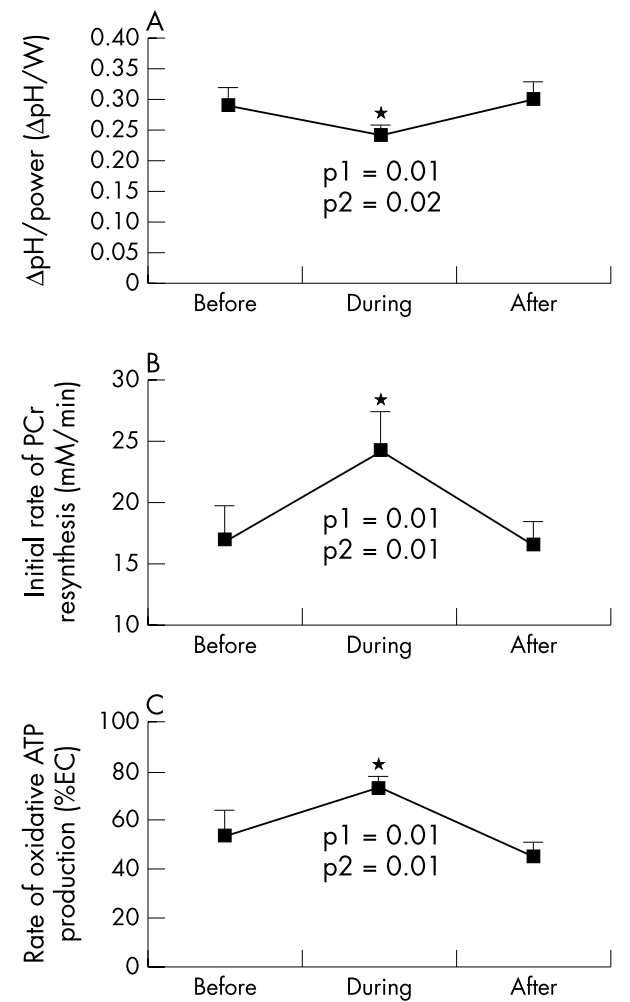

Figure 4 Changes in variables measured before, during, and after the treatment period (see text for definition). (A) $\Delta \mathrm{pH} /$ power refers to the $\mathrm{pH}$ changes recorded at end of the exercise period and scaled to the power output. (B) Initial rate of phosphocreatine (PCr) resynthesis $\left(\mathrm{Vi}_{\mathrm{rec}}\right)$ measured during the initial recovery period. (C) Changes in the rate of oxidative ATP production measured before, during, and after the treatment period expressed as percentage of energy cost (\%EC). Results are presented as means with error bars representing $1 \mathrm{SE}$. The asterisk indicates a significant difference compared with the measurements performed before ( 1$)$ and after ( $p 2$ ) the treatment period. Details on measurements performed before, during, and after treatment are specified in the text.

assuming that the recovery is monoexponential. Alternatively, assuming a biexponential model, some authors have used a semilog model using the first few points recorded after the end of exercise. ${ }^{28}$ We tested the two methods on our data (results not shown) and checked that they gave similar results-that is, an improvement in muscle oxidative function associated with CM treatment.

Several variables measured during exercise and recovery indicated improved oxidative metabolism after CM treatment, whereas no effect on resting variables was observed. The absence of a placebo control group meant that we could not eliminate a placebo effect, which would have to be addressed by a double blinded placebo versus control trial. However, this should be less of a problem than it may seem, given the convincing changes and stability of the measurements before treatment.

The reduction in $\Delta \mathrm{pH} /$ power measured at the end of exercise probably accounts for the decreased rate of anaerobic ATP production. Cytosolic pH changes in skeletal muscle partly reflect anaerobic ATP production, but are also modulated by PCr breakdown, proton efflux, and buffering components. We did not observe any changes in the amount of PCr used, the cytosolic buffering capacity, or kinetics of $\mathrm{pH}$ recovery associated with the reduction in $\Delta \mathrm{pH} /$ power, indicating that, for a given work rate, these limited $\mathrm{pH}$ changes reflect a reduced contribution of glycogenolysis to ATP production. Accordingly, $\mathrm{pH}$ measured at the end of exercise during $\mathrm{CM}$ treatment was no different from before and after treatment, whereas the work rate increased significantly. This improvement is further 
illustrated by the shift towards a higher work output (fig 3). It was observed even though the same load was lifted during exercise, and it can be associated with increased tolerance to exercise during $\mathrm{CM}$ treatment. Also, as the relation between mechanical performance and energy expenditure remained constant throughout the protocol, this decrease in anaerobic ATP production must be associated with increased ATP production from another pathway. In fact, significantly more aerobically produced ATP was measured during CM treatment, further confirming the transient enhancement of the oxidative processes.

It has been shown that, assuming allosteric control of aerobic ATP production by ADP, the rate of oxidative ATP production measured at the end of exercise should be similar to the rate of [PCr] recovery. ${ }^{10} \mathrm{In}$ agreement with this, we measured an accel eration of the rate of [ $\mathrm{PCr}$ ] recovery after exercise during $\mathrm{CM}$ treatment. This improvement further confirms the increased aerobic ATP production associated with CM treatment. Indices of $[\mathrm{PCr}]$ recovery have proved to be sensitive to changes in aerobic capacity resulting from training ${ }^{27}$ or muscle disorders. ${ }^{14}{ }^{16}$ The rate of [PCr] recovery can also be modulated by oxygen availability ${ }^{29}$ and $\mathrm{pH}$ reached at the end of exercise. ${ }^{9}$ Also, it has been shown in animal experiments that the kinetics of [PCr] recovery may be influenced by changes in mitochondrial content. ${ }^{30}$ In our study, because of the time scale, the increased aerobic ATP production is probably due to a change in mitochondrial function, as previously reported under conditions of hyperoxia. ${ }^{29}$ In addition, the increase in work rate recorded during exercise cannot account for the improvement in mitochondrial function, considering that it has been widely reported that neither the work output ${ }^{10}$ nor the amount of $\mathrm{PCr}$ consumed ${ }^{29}$ affects the kinetics of [ $\mathrm{PCr}$ ] recovery. Hence, diet supplementation with $\mathrm{CM}$ enhances the capacity of muscle to produce ATP aerobically.

Citrulline is involved in the urea cycle and allows detoxification of ammonia. In comparison with placebo, it has been shown that treatment with CM led to a significant increase in levels of bicarbonate, ornithine, arginine, and citrulline. ${ }^{5}$ Previous investigations of the metabolic effects of $\mathrm{CM}$ on whole body measurements have shown an increased rate of ammonia clearance with no effect on the extent of accumulation during bicycle exercise. ${ }^{3}$ This faster clearance has been interpreted as a direct effect of citrulline. In our case, it is unlikely that citrulline affects aerobic ATP production. It is more likely that malate, a TCA intermediate, affects aerobic ATP production through anaplerotic reactions. $M$ alate is dehydrogenated in the TCA cycle to oxaloacetate, the concentration of which is one of the most critical controls of the rate of aerobic ATP production. Also, it is transported through the inner mitochondrial membrane by a dicarboxylate transport system. Five of the eight TCA intermediates, including malate, are known to take part in ancillary reactions that can alter the concentrations of TCA intermediates, thereby affecting fluxes into and out the TCA cycle. ${ }^{31}$

Studies on heart muscle have shown that the anaplerotic flux of carbon into the TCA cycle is a normal part of cellular metabolism. Although less information is available for skel etal muscle, it is likely that anaplerosis plays a similar role in this tissue and functions to replenish the pool of TCA intermediates after their removal from the cycle. ${ }^{31}$ Previous studies in humans and animals have shown that the total pool in skeletal muscle increases severalfold very quickly at the onset of contraction..$^{32}$ Of the TCA intermediates, malate shows the largest relative and absolute change during exercise, typically accounting for $350 \%$ of the net increase in total pool size. ${ }^{33}{ }^{34}$ It has been suggested that this increase in TCA intermediates is required to attain high rates of ATP production through the TCA cycle. ${ }^{33} 35$ This increase in TCA intermediates would augment the overall rate of TCA cycle flux by substrate activation of other near equilibrium reactions such as the dehydrogena- tion of malate to oxal oacetate catalysed by malate dehydrogenase. Interestingly, the anaplerotic flux of carbon into the TCA cycle clearly exceeds the removal of TCA intermediates at the onset of exercise, whereas the process is reversed when exercise is prolonged. ${ }^{31}$ In keeping with the stimulating effect of TCA intermediates on the overall TCA flux, several authors have suggested that, in the absence of TCA intermediate increase, the flux through the TCA cycle would decrease, thereby accounting for, at least in part, the development of local muscle fatigue. ${ }^{33} 35$ The expansion of the TCA intermediate pool can therefore be regarded as a means of attaining higher rates of aerobic energy production, in agreement with our results showing that malate supplementation promotes a greater contribution of aerobic ATP production to total energy production. These results suggest that this hyperactivation of aerobic ATP production coupled to a reduction in anaerobic energy supply may contribute to the reduction in fatigue sensation reported by the subjects.

\section{APPENDIX}

At each minute of exercise, rates of ATP production from $\mathrm{PCr}$ hydrolysis $\left(P_{\text {ATP }}\right)$, glycogenolysis $\left(G_{\text {ATP }}\right)$, and oxidative processes $\left(\mathrm{O}_{\text {ATP }}\right)$ were calculated by the method of Kemp \& al. ${ }^{24}$

Rate of ATP production from PCr hydrolysis $\left(\mathrm{P}_{\text {ATP }}\right)$ The rate of ATP production from PCr hydrolysis was cal culated from the time dependent changes in $[\mathrm{PCr}]$ at each minute of exercise. This rate was scaled to the power output at each minute of exercise.

Rate of ATP production from glycogenolysis $\left(\mathrm{G}_{\mathrm{ATP}}\right)$

Throughout the exercise period, glycogen breakdown to pyruvate and lactate leads to a decrease in $\mathrm{pH}$, which is limited by proton efflux, the buffering power of cytosol, and PCr hydrolysis. Assuming that the glycogenolytic production of 1 ATP, when coupled to ATP hydrolysis, yields 1.5 protons, $G_{\text {ATP }}$ can be simply deduced from the total number of protons produced throughout exercise. ${ }^{36}$ This metabolic proton generation can be calculated from $\mathrm{pH}$ changes (measured by ${ }^{31} \mathrm{P} M \mathrm{RS}$ ), the number of protons passively buffered in the cytosol $\left(\left[\mathrm{H}^{+}\right] \beta\right.$; equation (1)), the number of protons consumed by net $\mathrm{PCr}$ hydrolysis ([ $\left.\mathrm{H}^{+}\right] \mathrm{PCr}$; equation (2)), and the number of protons leaving the cell (proton efflux; equation (3)).

$\left[\mathrm{H}^{+}\right] \beta=\beta \times \Delta \mathrm{pH}$

where

$\beta=\beta$ non-bicarbonate-non- $P_{i}+\beta P_{i}+\beta P M E+\beta$ bicarbonate

where

Bnon-bicarbonate-non- $P_{i}=(-22 \times \Delta p H)+170 .{ }^{26}$

$\beta \mathrm{P}_{\mathrm{i}}$ and $\beta \mathrm{PME}$ (phosphomonoester) were determined based on the dissociation constant of the buffer $(K)$ according to the standard formula ${ }^{25}$

$$
=\left(2.303\left[\mathrm{H}^{+}\right] \mathrm{K}[\mathrm{X}]\right) /\left(\mathrm{K}+\mathrm{H}^{+}\right)^{2}
$$

where $X$ is either $P_{i}$ or $P M E$ and $K=1.77 \times 10^{-7}$ for $P_{i}$ and 6.3 $\times 10^{-7}$ for $\mathrm{PM} \mathrm{E}$.

In agreement with previous studies and assuming that muscle is a closed system during exercise, ${ }^{25}{ }^{26} \beta$ bicarbonate was set to zero.

$\left[\mathrm{H}^{+}\right]_{\mathrm{PCr}}=\gamma \mathrm{V}_{\mathrm{PCr}}$ 


\section{Take home message}

The rate of muscle oxidative ATP production during exercise and the rate of $\mathrm{PCr}$ recovery after exercise both increase in subjects supplemented for 15 days with citrulline/ malate. This positive effect of citrulline/ malate at the oxidative level may be mediated by malate, a tricarboxylic acid cycle intermediate.

where $\gamma$ is the proton stoichiometric coefficient of the coupled Lohmann reaction, as described originally by Kushmerick, ${ }^{37}$ and $\mathrm{V}_{\mathrm{PCr}}$ is the rate of $\mathrm{PCr}$ consumption measured for each minute of exercise.

Throughout the recovery period, despite the intracellular proton load associated with $\mathrm{PCr}$ resynthesis $\left(\left[\mathrm{H}^{+}\right]_{\mathrm{Pr}}\right), \mathrm{pH}$ returns to its basal value as a result of proton efflux $(\mathrm{Pe})$. Hence, as reported by Kemp et al, ${ }^{26}$ proton efflux can be calculated assuming proton production from $\mathrm{PCr}$ resynthesis and $\mathrm{pH}$ changes are as follows:

$$
\operatorname{Pe}(\mathrm{mM} / \min )=\mathrm{V}_{\mathrm{pH}} \beta+\left[\mathrm{H}^{+}\right]_{\mathrm{PCr}}
$$

where $\beta$ represents the buffering capacity of muscle cytosol, $\mathrm{V}_{\mathrm{pH}}$ is the rate of $\mathrm{pH}$ change during the initial period of recovery, and $\left[\mathrm{H}^{+}\right]_{\mathrm{PC}}$ is the rate of proton production associated with $\mathrm{PCr}$ resynthesis. Taking into account changes in $\mathrm{pH}$ and $[\mathrm{PCr}]$ throughout the initial period of recovery, we have previously determined a linear relation between $\mathrm{Pe}$ and the extent of intracellular acidosis measured at end of exercise $(\Delta \mathrm{pH}) .^{38}$

Assuming, in agreement with others, ${ }^{10}$ that this $\mathrm{pH}$ dependence of proton efflux remains valid during exercise, proton efflux was calculated for each minute of exercise based on the $\Delta \mathrm{pH}$ calculated at the corresponding time of exercise.

Once $\mathrm{G}_{\text {ATP }}$ is calculated, it is scaled to the power output for each minute of exercise.

\section{Rate of anaerobic ATP production $\left(\mathrm{An}_{\mathrm{ATP}}\right)$ and energy} cost (EC)

The rate of anaerobic ATP production scaled to power output corresponds to the sum of $P_{\text {ATP }}+G_{\text {ATP }}$. It represents the amount of anaerobically produced ATP for a given unit of power. At the onset of exercise, it has been shown from a comparative analysis of ischaemic and aerobic exercise ${ }^{10}$ that the contribution of oxidative metabolism is minor compared with that of anaerobic metabolism. Under these conditions, the rate of anaerobic ATP production scaled to power output calculated at the onset of exercise can be considered a reliable estimation of the total rate of ATP production necessary for a given unit of power (the energy cost).

Rate of oxidative ATP production $\left(\mathrm{O}_{\text {ATP }}\right)$ Assuming that the energy cost remains proportional to power output throughout the exercise period, any decrease in anaerobic ATP production must be compensated for by an increase in oxidative ATP production, $\mathrm{O}_{\text {ATP. }}$. Therefore, $\mathrm{O}_{\text {ATP }}$ can be calculated at any time during exercise as the difference between EC and anaerobic ATP production scaled to power output.

Alternatively, the rate of oxidative ATP production at the end of exercise can be estimated from the initial rate of [PCr] recovery. ${ }^{39}$

\section{ACKNOWLEDGEMENTS}

This work was supported by grants from AFM (Association Française contre les Myopathies), PHRC 1997 (Programme Hospitalier de Recherche Clinique), and CNRS (Centre National de la Recherche Scientifique)
Authors' affiliations

D Bendahan, J P Mattei, S Confort-Gouny, PJ Cozzone, Centre de Résonance M agnétique Biologique et M édicale, UM R CN RS 6612,

Faculté de M édecine de la Timone, Marseille, France

B Ghattas, G roupement de Recherche en Econométrie Q uantitative d'Aix-M arseille, Marseille

M E Le Guern, Laboratoire Biocodex, 92120 M ontrouge, France

\section{REFERENCES}

1 Creff AF. Controlled double-blind clinical-study against stimol placebo in the treatment of asthenia. Gazette Medicale De France 1982;89:1926-9

2 Dauverchain J. Double-blind study of Stimol in the treatment of asthenia in older subjects. Mediterranée Médicale 1982:272:77-9.

3 Vanuxem D, Duflot JC, Prevot $H$, et al. Influence of an anti-asthenia agent, citrulline malate, on serum lactate and ammonia kinetics during a maximum exercise test in sedentary subjects. Séminaire des Hôpitaux de Paris 1990;66:477-81.

4 Fornaris E, Vanuxem D, Duflot JC, et al. Pharmacoclinical approach of citrulline malate activity: analysis of blood lactate during a standardised exercise. Gazette Medicale 1984;91:1-3

5 Callis A, Debornier BM, Serrano JJ, et al. Activity of citrulline malate on acid-base-balance and blood ammonia and amino-acid levels: study in the animal and in man. Arzneimittelforschung 1991:41:660-3.

6 Briand J, Astoin J, Lavalmartin D, et al. Euglena, as a cellular-model used in pharmacology for studying the effects of citrulline malate on lactate metabolisation. Comp Biochem Physiol 1986;85:553-8.

7 Goubel F, Vanhoutte C, Allaf 0 et al. Citrulline malate limits increase in muscle fatigue induced by bacterial endotoxins. Can I Physiol Pharmaco $1997 ; 75: 205-7$

8 Taylor DJ, Bore PJ, Styles $P$, et al. Bioenergetics of intact human muscle. A ${ }^{31} \mathrm{P}$ nuclear magnetic resonance study. Mol Biol M ed 1983;1:77-94.

9 Bendahan D, Confort-G ouny S, Kozak-Reiss G, et al. Heterogeneity of metabolic response to muscular exercise in humans: new criteria of invariance defined by in vivo P-31 NMR spectroscopy. FEBS Lett $1990 ; 272: 155-8$

10 Kemp GJ, Thompson CH, Barnes PRJ, et al. Comparisons of ATP turnover in human muscle during ischemic and aerobic exercise using P-31 magnetic resonance spectroscopy. Magn Reson Med $1994 ; 31: 248-58$

11 Kushmerick MJ. N ormal muscle energy metabolism. Adv Exp M ed Biol $1984 ; 178: 339-50$

12 McCully KK, Kent JA, Chance B. Application of ${ }^{31} \mathrm{P}$ magnetic resonance spectroscopy to the study of athletic performance. Sports Med 1988;5:312-21.

13 Kent-Braun JA, M cC ully KK, Chance B. Metabolic effects of training in humans: a ${ }^{31}$ P-M RS study. J Appl Physiol 1990;69:1165-70.

14 Bendahan D, Desnuelle C, Vanuxem D, et al. P-3 I N M R spectroscopy and ergometer exercise test as evidence for muscle oxidative performance improvement with coenzyme-Q in mitochondrial myopathies. N eurology 1992;42:1203-8

15 Lodi R, Taylor DJ, Tabrisi SJ, et al. N ormal in vivo skeletal muscle oxidative metabolism in sporadic inclusion body myositis assessed by P-31-magnetic resonance spectroscopy. Brain 1998;121:2119-26.

16 Taylor DJ, Kemp G J, Radda GK. Bioenergetics of skeletal-muscle in mitochondrial myopathy. I N eurol Sci 1994;127:198-206.

17 Mac Nelley GW. The development and laboratory validation of subjective fatigue scale. In: Tiffin J, M cC ormick EJ, eds. Industrial psychology. London: G eorges Allen and Unwin Ltd, 1966

18 Huskisson EC. M easurement of pain. Lancet 1974;9:1127-31.

19 Bendahan D, Confort-G ouny S, Kozak-Ribbens G, et al. 31-P N M R characterisation of the metabolic anomalies associated with the lack of glycogen-phosphorylase activity in human forearm muscle. Biochem Biophys Res Commun 1992;185:16-21

20 Mazzeo AR, Levy GC. An evaluation of new processing protocols for in vivo N MR spectroscopy. Magn Reson Med 1991;17:483-95

21 Harris RC, Hultman E, N ordesjo LO. G lycogen, glycolytic intermediates and highenergy phosphates determined in biopsy samples of musculus quadriceps femoris of man at rest. $M$ ethods and variance of values. Scand J Clin Lab Invest 1974;33:109-20.

22 Moon RB, Richards JH. Determination of intracellular $\mathrm{pH}$ by ${ }^{31} \mathrm{P}$ magnetic resonance. I Biol Chem 1973:248:7276-8.

23 McCully KK, lotti S, Kendrick K, et al. Simultaneous in vivo measurements of $\mathrm{HbO}_{2}$ saturation and $\mathrm{PCr}$ kinetics after exercise in normal humans. J Appl Physiol 1994;77:5-10

24 Kemp GJ, Thompson CH, Taylor DJ, et al. ATP production and mechanical work in exercising skeletal muscle: a theoretical analysis applied to ${ }^{31} \mathrm{P}$ magnetic resonance spectroscopic studies of dialyzed uremic patients. Magn Reson Med 1995;33:601-9.

25 Conley KE, Kushmerick MJ, Jubrias SA. G lycolysis is independent of oxygenation state in stimulated human skeletal muscle in vivo. J Physiol (Lond) 1998:511:935-45

26 Kemp GJ, Taylor DJ, Styles P, et al. The production, buffering and efflux of protons in human skeletal muscle during exercise and recovery. NMR Biomed 1993;6:73-83.

27 McCully KK, Boden BP, Tuchler M, et al. W rist flexor muscles of elite rowers measured with magnetic resonance spectroscopy. J Appl Physiol $1989 ; 67: 926-32$ 
28 Taylor DJ, Kemp G J, Radda GK. Bioenergetics of skeletal muscle in mitochondrial myopathy. J N eurol Sci 1994;127:198-206

29 Haseler LJ, Hogan M C, Richardson RS. Skeletal muscle phosphocreatine recovery in exercise-trained humans is dependent on $0-2$ availability. J Appl Physiol 1999:86:2013-18.

30 Paganini AT, Foley JM, M eyer RA. Linear dependence of muscle phosphocreatine kinetics on oxidative capacity. A m J Physiol 1997;272:C501-10.

31 Gibala MJ, Young ME, Taegtmeyer H. A naplerosis of the citric acid cycle: role in energy metabolism of heart and skeletal muscle. Acta Physiol Scand 2000;168:657-65.

32 Gibala MJ, Tarnopolsky M A, G raham TE. Tricarboxylic acid cycle intermediates in human muscle at rest and during prolonged cycling. Am J Physiol 1997;35:E2 39-44.

33 Sahlin K, Katz A, Broberg S. Tricarboxylic acid cycle intermediates in human muscle during prolonged exercise. [Published errata in $A \mathrm{~m}$ ] Physiol 1995;268(2 pt 1):section C following table of contents and $1995 ; 268(6$ pt 3):section C following table of contents.] Am J Physiol $1990 ; 259(5$ pt 1):C834-41.
34 Gibala MJ, MacLean DA, G raham TE, et al. Tricarboxylic acid cycle intermediate pool sise and estimated cycle flux in human muscle during exercise. Am J Physiol 1998;275(2 pt 1):E235-42.

35 Wagenmakers AJ. Muscle a mino acid metabolism at rest and during exercise: role in human physiology and metabolism. Exerc Sport Sci Rev 1998;26:287-314.

36 Hochachka PW, Mommsen TP. Protons and anaerobiosis. Science 1983;219:1391-7

37 Kushmerick MJ. Multiple equilibria of cations with metabolites in muscle bioenergetics. Am J Physiol 1997;272(5 pt 1):C1739-47.

38 Vialettes $B$, Paquisfluckinger V, Silvestreaillaud $P$, et al. Extra-pancreatic manifestations in diabetes secondary to mitochondrial DNA point mutation within the tRN A (Leu(Uur)) gene. Diabetes Care 1995;18:1023-8.

39 Boska M. Estimating the ATP cost of force production in the human gastrocnemius/ soleus muscle group using ${ }^{31} \mathrm{P} M \mathrm{MS}$ and ${ }^{1} \mathrm{H} \mathrm{MRI}$. N MR Biomed 1991;4:173-81.

\section{EDUCATIO N PRO GRAM ME}

\section{British A ssociation of Sport and Exercise Medicine in association with the $\mathrm{N}$ ational Sports Medicine Institute}

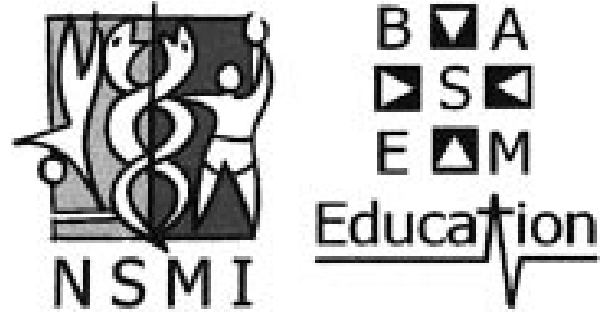

Education programme 2002

General Sports Medicine

Lilleshall National Sports Centre, 22-27 September

Practical Sport and Medicine Meeting

Club La Santa, Lanzarote (families \& non-delegates welcome), 3-10 October

Diploma Preparation

Location and date to be confirmed, October

The $\mathrm{Q}$ ueen's $\mathrm{G}$ olden Jubilee \& Post Commonwealth $\mathrm{G}$ ames BA SEM Congress

The Low Wood Hotel and Conference Centre, Windermere,

10-13 October
Intermediate Sports Injury M anagement and M edicine- Lower Limb Lilleshall National Sports Centre, 17-22 November

Current Concepts

Topic, location, and date to be confirmed, December

Education programme 2003

Intermediate Sports Injury M anagement and M edicine-Head, N eck, and Upper Limb

Lilleshall National Sports Centre, 23-28 February

General Sports Medicine

Lilleshall National Sports Centre, 27 A pril-2 M ay Diploma Preparation Course

Sheffield Centre of Sports M edicine, M ay

Current Concepts

Topic, location, and date to be confirmed, M ay

Intermediate Sports Injury M anagement and M edicine- Lumbar

Spine, Thorax, Groin, Pelvis, and Hip

Lilleshall National Sports Centre, 6-11 J uly

General Sports M edicine

Lilleshall National Sports Centre, 21-26 September

For further details of these courses please contact $\mathrm{M} \mathrm{r}$ Barry Hill, The National Sports Medicine Institute, 32 Devonshire Street, London W1G 6PX, UK. Tel: 0207908 3642; Fax: 0207908 3635; email: barry.hill@nsmi.org.uk; www.nsmi.org.uk 